\section{(6) OPEN ACCESS}

\title{
Comprehensive characterisation of hypertensive heart disease left ventricular phenotypes
}

\author{
Jonathan C L Rodrigues, ${ }^{1,2}$ Antonio Matteo Amadu, ${ }^{1,3}$ Amardeep Ghosh Dastidar, ${ }^{1,4}$ \\ Gergley V Szantho, ${ }^{1,5}$ Stephen M Lyen, ${ }^{1,6}$ Cattleya Godsave, ${ }^{7}$ Laura E K Ratcliffe, ${ }^{8}$ \\ Amy E Burchell, ${ }^{4,8}$ Emma C Hart, ${ }^{2,8}$ Mark C K Hamilton, ${ }^{6}$ Angus K Nightingale, ${ }^{4,8}$ \\ Julian F R Paton, ${ }^{2,8}$ Nathan E Manghat, ${ }^{1,6}$ Chiara Bucciarelli-Ducci ${ }^{1,4}$
}

\begin{abstract}
- Additional material is published online only. To view please visit the journal online (http://dx.doi.org/10.1136/ heartjnl-2016-309576).

For numbered affiliations see end of article.

\section{Correspondence to} Dr Chiara Bucciarelli-Ducci, NIHR Bristol Cardiovascular Biomedical Research Unit, Bristol Heart Institute, University Hospitals Bristol NHS Foundation Trust, Upper Maudlin Street, Bristol BS2 8HW, UK; C.Bucciarelli-Ducci@ bristol.ac.uk
\end{abstract}

Received 2 March 2016 Revised 29 April 2016 Accepted 8 May 2016 Published Online First 3 June 2016

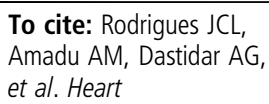

\section{ABSTRACT}

Objective Myocardial intracellular/extracellular structure and aortic function were assessed among hypertensive left ventricular (LV) phenotypes using cardiovascular magnetic resonance (CMR).

Methods An observational study from consecutive tertiary hypertension clinic patients referred for CMR (1.5 T) was performed. Four LV phenotypes were defined: (1) normal with normal indexed LV mass (LVM) and LVM to volume ratio (M/V), (2) concentric remodelling with normal LVM but elevated $M / V_{\text {, }}$ (3) concentric LV hypertrophy (LVH) with elevated LVM but normal indexed end-diastolic volume (EDV) or (4) eccentric LVH with elevated LVM and EDV. Extracellular volume fraction was measured using T1-mapping. Circumferential strain was calculated by voxel-tracking. Aortic distensibility was derived from high-resolution aortic cines and contemporaneous blood pressure measurements.

Results 88 hypertensive patients $(49 \pm 14$ years, $57 \%$ men, systolic blood pressure (SBP): $167 \pm 30 \mathrm{~mm} \mathrm{Hg}$, diastolic blood pressure (DBP): $96 \pm 14 \mathrm{~mm} \mathrm{Hg}$ ) were compared with 29 age-matched/sex-matched controls (47 \pm 14 years, $59 \%$ men, SBP: $128 \pm 12 \mathrm{~mm} \mathrm{Hg}$, DBP: $79 \pm 10 \mathrm{~mm} \mathrm{Hg}$ ). LVH resulted from increased myocardial cell volume (eccentric LVH: $78 \pm 19 \mathrm{~mL} / \mathrm{m}^{2}$ vs concentric LVH: $73 \pm 15 \mathrm{~mL} / \mathrm{m}^{2}$ vs concentric remodelling: $55 \pm 9 \mathrm{~mL} / \mathrm{m}^{2}$, $\mathrm{p}<0.05$, respectively) and interstitial fibrosis (eccentric LVH: $33 \pm 10 \mathrm{~mL} / \mathrm{m}^{2}$ vs concentric LVH: $30 \pm 10 \mathrm{~mL} / \mathrm{m}^{2}$ vs concentricremodelling: $19 \pm 2 \mathrm{~mL} / \mathrm{m}^{2}, p<0.05$, respectively). LVH had worst circumferential impairment (eccentric LVH: $-12.8 \pm 4.6 \%$ vs concentric LVH: -15.5 $\pm 3.1 \%$ vs concentric remodelling: $-17.1 \pm 3.2 \%$, $\mathrm{p}<0.05$, respectively). Concentric remodelling was associated with reduced aortic distensibility, but not with large intracellular/interstitial expansion or myocardial dysfunction versus controls.

Conclusions Myocardial interstitial fibrosis varies across hypertensive LV phenotypes with functional consequences. Eccentric LVH has the most fibrosis and systolic impairment. Concentric remodelling is only associated with abnormal aortic function. Understanding these differences may help tailor future antihypertensive treatments.

\section{INTRODUCTION}

Hypertensive left ventricular hypertrophy (LVH) is an independent predictor of sudden cardiac death ${ }^{1}$ and heart failure. ${ }^{2}$ International hypertension guidelines $^{3}$ highlight its prognostic importance. However, hypertensive left ventricular (LV) phenotypes can be further classified as: normal structure (normal LV mass (LVM) and relative wall thickness), concentric remodelling (normal LVM but elevated relative wall thickness) and $\mathrm{LVH}^{4}$ Similar classifications can be made with cardiovascular magnetic resonance (CMR), using mass to volume ratio $(\mathrm{M} / \mathrm{V})$ in lieu of relative wall thickness. ${ }^{5}$ Hypertensive LVH and concentric remodelling are both predictors of cardiovascular morbidity and mortality. ${ }^{6}$ However, their pathophysiology is incompletely understood.

Diffuse interstitial myocardial fibrosis has been documented histologically in hypertension. ${ }^{7}$ Precontrast (native) T1-mapping is a non-invasive CMR technique that quantifies changes in myocardial intracellular and/or extracellular compartments. ${ }^{8}$ In conjunction with postcontrast T1-mapping, myocardial extracellular volume fraction (ECV) can be calculated to localise abnormality to the interstitium. T1-mapping quantification of interstitial fibrosis has been validated against histological gold standard. ${ }^{9}$

We investigated whether differences exist between hypertensive LV phenotypes in myocardial intracellular/extracellular structure and myocardial/ aortic function with CMR using T1-mapping and voxel-tracking techniques. We hypothesised that the incremental adverse prognosis of LVH over the other hypertensive phenotypes would correlate with the burden of diffuse myocardial fibrosis and myocardial dysfunction.

\section{MATERIALS AND METHODS \\ Study population}

An observational study from consecutive tertiary hypertension clinic patients referred for CMR (1.5 T) between February 2012 and April 2015 was performed. The study conformed to governance arrangements for research ethics committees. Subjects provided written consent. Baseline demographic and clinical characteristics were recorded. Exclusion criteria were any concomitant myocardial pathology that may confound hypertrophy (eg, moderate-severe valvular disease, acquired/inherited cardiomyopathy) and estimated glomerular filtration rate $<30 \mathrm{~mL} / \mathrm{min} / 1.73 \mathrm{~m}^{2}$. Normotensive healthy volunteers were enrolled as controls.

Average office systolic blood pressure (SBP) and diastolic blood pressure (DBP) were acquired after 
seated rest from both arms using standard automated sphygmomanometry. Patients were stratified by severity in accordance with European guidelines. ${ }^{3}$

\section{CMR cine protocol and analysis}

CMR was performed at $1.5 \mathrm{~T}$ (Avanto, Siemens, Germany). Short-axis steady-state free precession (SSFP) cines with whole LV coverage $(8 \mathrm{~mm}$ slice thickness, no slice gap, temporal resolution $38.1 \mathrm{~ms}$, echo time $1.07 \mathrm{~ms}$, in-plane pixel size $1.5 \times 0.8 \mathrm{~mm}$ ) were used for estimating LVM and volumes, which

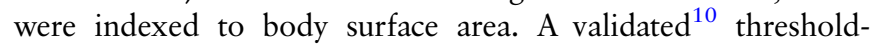
detection software (CMR42, Circle Cardiovascular Imaging, Canada) was employed to include papillary muscles/trabeculae in LVM and then include them in blood pool for volume measurements as before. ${ }^{11}$ LV dilatation and LVH were defined as indexed end-diastolic volume (EDV) and indexed LVM $>95$ th centile of age-specific and gender-specific CMR reference ranges. ${ }^{11}$ Increased $\mathrm{M} / \mathrm{V}$ was defined as $>95$ th confidence interval $(>1.16 \mathrm{~g} / \mathrm{mL})$ from previously reported data from 91 healthy volunteers. ${ }^{5}$ The mean $\mathrm{M} / \mathrm{V}$ in our study, using this cut-off value was $0.89 \pm 0.13 \mathrm{~g} / \mathrm{mL}$, which is consistent with the previous reported normal values of $0.88 \pm 0.14 \mathrm{~g} / \mathrm{mL} .^{5}$ Four hypertensive LV phenotypes were defined similar to previous echocardiographic $^{4}$ and CMR studies, ${ }^{5}$ according to LVM, EDV and M/V as: normal LV (normal indexed LVM and M/V), concentric remodelling (normal indexed LVM but increased M/V), concentric LVH (elevated indexed LVM but normal EDV) and eccentric LVH (elevated indexed LVM and EDV) (table 1). CMR analysis was performed by an experienced, blinded CMR reader.

\section{CMR late gadolinium protocol and analysis}

Replacement myocardial fibrosis was assessed by late gadolinium enhancement (LGE). Inversion recovery sequences were performed $10-15 \mathrm{~min}$ after intravenous $0.1 \mathrm{mmol} / \mathrm{kg}$ gadobutrol (Gadovist, Bayer Pharma AG, Germany). Inversion time was optimised to achieve myocardial nulling. LGE was assessed visually by consensus between two expert CMR readers, blinded to all other data. Patients with LGE were excluded to avoid confounding effects of myocardial replacement fibrosis.

\section{CMR T1-mapping protocol and analysis}

Myocardial T1-mapping was performed using the modified look-locker inversion recovery sequence $\left(35^{\circ}\right.$ flip angle, $100 \mathrm{~ms}$ minimum TI, $80 \mathrm{~ms}$ TI increment, $150 \mathrm{~ms}$ time delay with 5-(3)-3 heartbeat acquisition scheme). ${ }^{12}$ Using Argus software (Siemens, Germany), regions of interest were drawn within midseptum on short-axis, motion-corrected, native T1-maps and copied onto corresponding postcontrast maps, adjusting for partial-voluming and/or artefact, as previously described. ${ }^{13}$ Analysis was performed by an experienced blinded CMR reader.

Table 1 Cardiovascular magnetic resonance definitions of patterns of hypertensive heart disease left ventricular (LV) phenotypes

\begin{tabular}{llll}
\hline & $\begin{array}{l}\text { Indexed LVM } \\
\left(\mathrm{g} / \mathrm{m}^{2}\right)\end{array}$ & $\begin{array}{l}\text { Indexed EDV } \\
\left(\mathrm{mL} / \mathrm{m}^{2}\right)\end{array}$ & $\begin{array}{l}\mathrm{M} / \mathrm{V} \\
(\mathrm{g} / \mathrm{mL})\end{array}$ \\
\hline Normal LV & Normal & Normal & Normal \\
Concentric remodelling & Normal & $\downarrow$ & $\uparrow$ \\
Concentric LVH & $\uparrow$ & Normal & $\uparrow$ \\
Eccentric LVH & $\uparrow$ & $\uparrow$ & Normal \\
\hline EDV, end-diastolic volume; LVH, left ventricular hypertrophy; LVM, LV mass; LVM and \\
EDV are indexed to body surface area; M/V=mass:volume ratio.
\end{tabular}

\section{Aortic stiffness}

As previously described, ${ }^{17}$ measures of ascending aortic stiffness were measured as follows:

(i) distensibility $=\Delta \mathrm{A} /\left(\mathrm{A}_{\text {diast }} \times \Delta \mathrm{P}\right)$ and (ii) compliance $=\Delta \mathrm{A} / \Delta \mathrm{P}$ where:

- $\Delta \mathrm{A}\left(\mathrm{mm}^{2}\right)$ was defined $\mathrm{A}_{\text {syst }}-\mathrm{A}_{\text {diast }}$.

- $\mathrm{A}_{\text {syst }}$ is the ascending aortic area, measured from cine image perpendicular to the vessel at the level of the right pulmonary artery, at end-systole.

- $\mathrm{A}_{\text {diast }}$ is the ascending aortic area at end-diastole and $\Delta \mathrm{P}$ $(\mathrm{mm} \mathrm{Hg})$ is the pulse pressure estimated from SBP-DBP at time of CMR.

Measurements were acquired by an experienced blinded CMR reader. Excellent reproducibility of these measures has previously been reported. ${ }^{17}$

\section{Statistical analysis}

Statistical analysis was performed using SPSS V.21 (Armonk, New York, USA: IBM Corp.). Categorical variables were analysed using Fisher's exact test. Data are expressed as mean \pm SD where appropriate. Normally distributed continuous variables were compared using one-way analysis of variance with Bonferroni correction for multiple comparisons. Continuous variables that were not normally distributed were compared by Kruskal-Wallis tests. R-values quoted are for Pearson's correlation coefficient. Post hoc multiple linear regression was used to control for covariates of age, gender, body mass index, diabetes, SBP, DBP and number of antihypertensive medications, which were significantly different between some hypertensive LV phenotypes, on the T1-mapping, myocardial strain and aortic data. Significance was defined as two-tailed $\mathrm{p}<0.05$, where $\mathrm{p}$ values presented include Bonferroni adjustment for multiple comparisons where appropriate. 


\section{RESULTS}

\section{Demographics}

Of the 108 hypertensive subjects initially recruited who underwent CMR, 20 were excluded (figure 1), with six excluded because of LGE (three had mid-wall fibrosis and ancillary CMR features of hypertrophic cardiomyopathy, two had ischaemic LGE and one had subepicardial LGE suggesting previous myocarditis). The final hypertensive sample size was 88 (age: 49 \pm 14 years, men: $57 \%$, office SBP: $167 \pm 30 \mathrm{~mm} \mathrm{Hg}$, office DBP: $96 \pm 14 \mathrm{~mm} \mathrm{Hg}$ ). Twenty-nine healthy control subjects were recruited (age: $47 \pm 14$ years, men: 59\%, office SBP: 128 $\pm 12 \mathrm{~mm} \mathrm{Hg}$, office DBP: $79 \pm 10 \mathrm{~mm} \mathrm{Hg}$ ). Diabetes prevalence was similar between hypertensive subgroups (table 2 ).

\section{Findings in eccentric LVH}

Eccentric LVH was present in 9\% $(n=8)$. Subjects with eccentric LVH had the most advanced hypertrophy, with indexed LVM $\left(122 \pm 30 \mathrm{~g} / \mathrm{m}^{2}\right)$ higher than those classified as concentric LVH $\left(108 \pm 24 \mathrm{~g} / \mathrm{m}^{2}\right)$, concentric remodelling $\left(75 \pm 10 \mathrm{~g} / \mathrm{m}^{2}\right)$ and normal LV $\left(70 \pm 9 \mathrm{~g} / \mathrm{m}^{2}\right)$ (table 3$)$. Indexed LVM correlated positively, albeit weakly, with native $\mathrm{T} 1(\mathrm{R}=0.352, \mathrm{p}=0.001)$ and ECV $(\mathrm{R}=0.318, \mathrm{p}=0.003$ ) (see online supplementary figure S1A, B). The elevated native T1 and ECV values for eccentric LVH compared with the other subgroups persisted after correction for covariates (table 4). The elevated indexed LVM in eccentric LVH was a result of increases in both intracellular and interstitial myocardial components (figure 2).

There was a weak positive correlation between SBP and native $\mathrm{T} 1$ that was statistically significant $(\mathrm{R}=0.267, \mathrm{p}=0.012)$. There were no significant correlations between SBP and ECV $(\mathrm{R}=0.143, \mathrm{p}=0.185), \mathrm{DBP}$ and native $\mathrm{T} 1(\mathrm{R}=0.089, \mathrm{p}=0.411)$ or DBP and ECV $(R=-0.112, p=0.300)$ (see online supplementary figure $\mathrm{S} 2 \mathrm{~A}-\mathrm{D})$.
Eccentric LVH had the lowest peak circumferential strain values $(-12.8 \pm 4.6 \%)$ compared with concentric LVH $(-15.5$ $\pm 3.1 \%)$, concentric remodelling $(-17.1 \pm 3.2 \%)$, normal LV $(-17.6 \pm 3.0 \%)$ and controls $(-17.4 \pm 2.6 \%)$, with evidence of both systolic and diastolic strain impairment (table 3 ). These associations persisted after correction for covariates (table 4).

\section{Findings in concentric LVH}

Concentric LVH was present in $27 \%(\mathrm{n}=24)$ and associated with elevated indexed LVM $\left(108 \pm 24 \mathrm{~g} / \mathrm{m}^{2}\right)$ compared with concentric remodelling $\left(75 \pm 10 \mathrm{~g} / \mathrm{m}^{2}\right)$, normal LV $\left(70 \pm 9 \mathrm{~g} / \mathrm{m}^{2}\right)$ and controls $\left(61 \pm 11 \mathrm{~g} / \mathrm{m}^{2}\right)$. Concentric LVH subjects had elevated native T1 values $(1054 \pm 41 \mathrm{~ms})$ compared with those with LV remodelling $(1029 \pm 45 \mathrm{~ms})$, normal LV $(1031 \pm 35 \mathrm{~ms})$ and controls $(1024 \pm 41 \mathrm{~ms})$ (table 3$)$. These findings for native T1 and ECV persisted after correction for covariates (table 4). The increased indexed LVM in concentric LVH compared with subjects with concentric remodelling and normal LV was due to expansion of both the myocardial interstitium and the myocardial cell volume (figure 2).

Myocardial functional changes accompanied the changes in myocardial structure in concentric LVH, with lower peak circumferential strain values $(-15.5 \pm 3.1 \%)$ compared with concentric remodelling $(-17.1 \pm 3.2 \%)$, normal LV $(-17.6 \pm 3.0 \%)$ and controls $(-17.4 \pm 2.6 \%)$ despite no statistically significant differences in LV ejection fraction (table 3 ).

\section{Findings in concentric remodelling}

Subjects with concentric remodelling had no differences in SBP, DBP or prevalence of European Society of Hypertension (ESH)/ European Society of Cardiology (ESC) grade 3 blood pressure (BP) compared with other hypertensive subgroups (table 2). Concentric remodelling was not associated with large

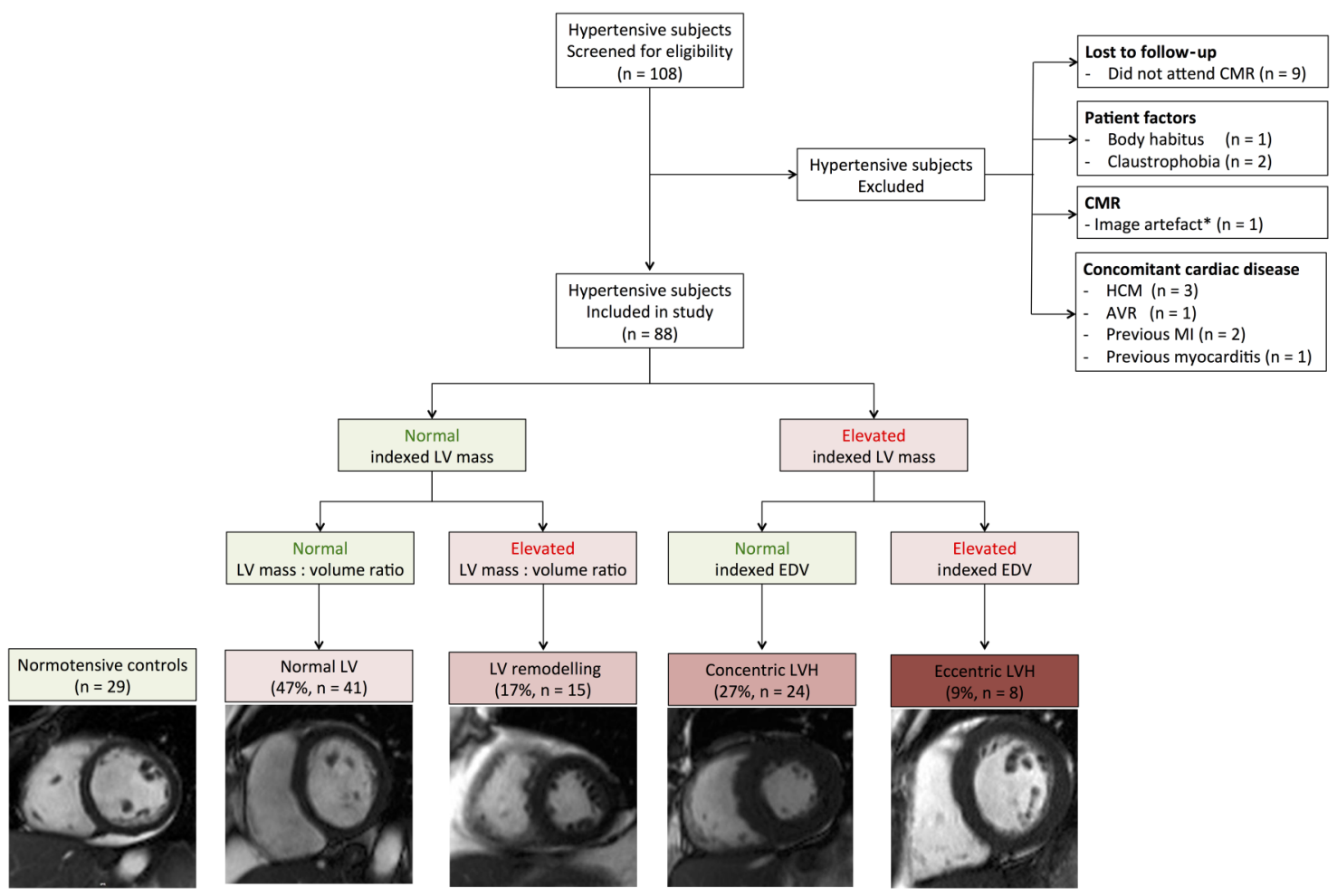

Figure 1 Study size and exclusion criteria. AVR, aortic valve replacement; CMR, cardiovascular magnetic resonance; EDV, end-diastolic volume; HCM, hypertrophic cardiomyopathy; LV, left ventricular; LVH, left ventricular hypertrophy; MI, myocardial infarction. *Image artefact from implantable loop recorder device precluding volumetric analysis. 
Table 2 Demographic data for hypertensive subjects and normotensive controls

\begin{tabular}{|c|c|c|c|c|c|}
\hline & \multirow[b]{3}{*}{ Controls $(n=29)$} & \multicolumn{4}{|c|}{ Hypertensive subjects ( $n=88$ ) } \\
\hline & & \multicolumn{2}{|c|}{ Normal indexed LVM $(n=56)$} & \multicolumn{2}{|c|}{ Elevated indexed LVM $(n=32)$} \\
\hline & & Normal LV ( $n=41)$ & Concentric remodelling $(n=15)$ & Concentric LVH $(n=24)$ & Eccentric LVH $(n=8)$ \\
\hline Age (years) & $47 \pm 13$ & $45 \pm 16$ & $56 \pm 12^{* 1}$ & $48 \pm 12$ & $56 \pm 11 * 2$ \\
\hline Gender ( $\%$ male $)$ & 59 & $41 * 3$ & 60 & 71 & 88 \\
\hline \multicolumn{6}{|l|}{ Ethnicity } \\
\hline Caucasian & $93^{* 4}$ & 73 & 93 & 83 & 87 \\
\hline Black African & 3 & 0 & 0 & 9 & 13 \\
\hline Black Caribbean & 0 & 10 & 0 & 4 & 0 \\
\hline Oriental & 0 & 2 & 0 & 0 & 0 \\
\hline South East Asian & 3 & 12 & 0 & 0 & 0 \\
\hline Mixed & 0 & 2 & 7 & 4 & 0 \\
\hline BMI $\left(\mathrm{kg} / \mathrm{m}^{2}\right)$ & $26 \pm 5 * 5$ & $30 \pm 6$ & $33 \pm 5$ & $31 \pm 6$ & $32 \pm 7$ \\
\hline Diabetes (\%) & $0^{* 6}$ & 5 & 13 & 17 & 20 \\
\hline Heart rate (bpm) & $67 \pm 12$ & $72 \pm 12$ & $76 \pm 16^{* 7}$ & $68 \pm 9$ & $65 \pm 13$ \\
\hline Office SBP (mm Hg) & $128 \pm 12^{* 8}$ & $161 \pm 26$ & $175 \pm 33$ & $170 \pm 30$ & $172 \pm 37$ \\
\hline Office DBP ( $\mathrm{mm} \mathrm{Hg}$ ) & $79 \pm 10 * 8$ & $94 \pm 11$ & $94 \pm 15$ & $99 \pm 14$ & $102 \pm 21$ \\
\hline \multicolumn{6}{|l|}{ ESH/ESC office BP grade } \\
\hline Controlled (\%) & $\ldots$ & 5 & 7 & 13 & 10 \\
\hline High normal (\%) & $\ldots$ & 7 & 7 & 0 & 0 \\
\hline Grade $1(\%)$ & $\ldots$ & 39 & 13 & 25 & 10 \\
\hline Grade $2(\%)$ & $\ldots$ & 22 & 27 & 29 & 30 \\
\hline Grade $3(\%)$ & $\ldots$ & 22 & 47 & 33 & 50 \\
\hline Isolated systolic HTN (\%) & $\ldots$ & 5 & 7 & 0 & 0 \\
\hline No. antihypertensive medications & $0^{\star 8}$ & $2 \pm 1$ & $2 \pm 2$ & $3 \pm 2 * 9$ & $4 \pm 3 * 10$ \\
\hline ACEi/ARB $(\%)$ & $0^{\star 8}$ & 68 & 80 & 83 & $100 * 11$ \\
\hline
\end{tabular}

${ }^{* 1}$ Concentric remodelling versus Normal LV: $p=0.007$ and Concentric remodelling versus Controls: $p=0.037$.

${ }^{* 2}$ Eccentric LVH versus Normal LV: $p=0.035$.

${ }^{*}$ Normal LV versus Concentric LVH: $p=0.020$ and Normal LV versus Eccentric LVH: $p=0.016$

${ }^{* 4}$ Controls versus Normal LV: $p=0.027$.

${ }^{*}$ Controls versus Normal LV: $p=0.017$, Controls versus Concentric remodelling: $p<0.0001$, Controls versus Concentric LVH: $p=0.001$ and Controls versus Eccentric LVH: $p=0.009$.

${ }^{* 6}$ Controls versus Concentric LVH: $p=0.030$ and Controls versus Eccentric LVH: $p=0.025$.

${ }^{* 7}$ Concentric remodelling versus Controls: $p=0.032$, Concentric remodelling versus Concentric LVH: $p=0.043$ and Concentric remodelling versus Eccentric LVH: $p=0.041$.

${ }^{* 8}$ Controls versus Normal LV: $p<0.0001$, Controls versus Concentric remodelling: $p<0.0001$, Controls versus Concentric LVH: $p<0.0001$ and Controls versus Eccentric LVH: $p<0.0001$

${ }^{* 9}$ Concentric LVH versus Normal LV: $p=0.011$.

${ }^{* 10}$ Eccentric LVH versus Concentric remodelling $p=0.016$ and Eccentric LVH versus Normal LV: $p=0.001$

*11Eccentric LVH versus Normal LV: $p=0.035$.

European Society of Hypertension/European Society of Cardiology (ESH/ESC) Office BP grade-Controlled SBP: 120-129 and/or DBP 80-84, High normal SBP: 130-139 mm Hg and/or DBP $85-89$ mm Hg, Grade 1 SBP 140-159 and/or DBP 90-99, Grade 2 SBP 160-179 and/or DBP 100-109, Grade 3 SBP $\geq 180$ and/or DBP $\geq 110$, Isolated systolic hypertension SBP

$\geq 140 \mathrm{~mm} \mathrm{Hg}$ and DBP $<90 \mathrm{~mm} \mathrm{Hg}$.

ACEi, angiotensin converting enzyme inhibitor; ARB, angiotensin II receptor blocker; BP, blood pressure; BMI, body mass index; DBP, diastolic blood pressure; HTN, hypertension; LV, left ventricular; LVH, left ventricular hypertrophy; SBP, systolic blood pressure.

intracellular/extracellular myocardial changes compared with controls (native T1 $1031 \pm 35$ vs $1024 \pm 41 \mathrm{~ms}, \mathrm{p}=0.465$ ). There were no large differences in myocardial strain in concentric remodelling compared with controls (table 3). Aortic compliance and distensibility were reduced in all hypertensive phenotypes, including normal LV, compared with controls. However, concentric remodelling was associated with the lowest aortic distensibility and compliance (tables 3 and 4).

\section{Determinants of myocardial systolic strain}

Mean circumferential strain data are demonstrated in figure 3. Increasing indexed myocardial cell volume $(\mathrm{R}=0.507$, $\mathrm{p}<0.0001)$ and increasing indexed interstitial volume $(\mathrm{R}=0.452, \mathrm{p}<0.0001)$ both correlated with worsening peak circumferential strain (figure 4). As there was significant difference in circumferential strain between concentric and eccentric LVH, post hoc analysis with a one-way analysis of covariance (ANCOVA) was conducted to determine whether the concomitant differences in indexed LVM and/or indexed EDV were responsible. The predicted main effect of indexed LVM was significant, $F(2,29)=12.3, p=0.002$, but that of indexed EDV was not significant, $F(2,29)=0.25, p=0.621$. Increased LVM is the likely reason for impaired circumferential strain in eccentric LVH compared with concentric LVH and this is consistent with our other results demonstrating significant positive correlations of both indexed myocardial cell volume and interstitial volume with peak circumferential strain.

\section{DISCUSSION}

This study investigates changes at the intracellular/extracellular myocardial structural level between the different hypertensive heart disease phenotypes and investigates whether such changes are associated with myocardial and aortic functional consequences. We show that: (i) hypertensive LVH is associated with elevated indexed LVM due to significant expansion of the interstitium as well as the myocardial cell component. It is associated with significant systolic and diastolic circumferential strain impairment. These significant findings occur both in eccentric LVH and concentric LVH but are most advanced in the former. (ii) In hypertensive concentric remodelling, there was no large 
Table 3 Cardiovascular magnetic resonance volumetric, T1-mapping and myocardial strain data for hypertensive subjects and normotensive controls

\begin{tabular}{|c|c|c|c|c|c|}
\hline & \multirow[b]{3}{*}{ Controls $(n=29)$} & \multicolumn{4}{|c|}{ Hypertensive subjects $(n=88)$} \\
\hline & & \multicolumn{2}{|c|}{ Normal indexed LVM $(n=56)$} & \multicolumn{2}{|c|}{ Elevated indexed LVM $(n=32)$} \\
\hline & & Normal LV $(n=41)$ & Concentric remodelling $(n=15)$ & Concentric LVH $(n=24)$ & Eccentric LVH $(n=8)$ \\
\hline \multicolumn{6}{|l|}{ LV volumetrics } \\
\hline Ejection fraction (\%) & $66 \pm 7$ & $67 \pm 7$ & $73 \pm 7^{* 1}$ & $67 \pm 7$ & $54 \pm 15^{\star 2}$ \\
\hline Indexed EDV $\left(\mathrm{mL} / \mathrm{m}^{2}\right)$ & $77 \pm 18$ & $76 \pm 12$ & $55 \pm 8^{* 3}$ & $81 \pm 11$ & $109 \pm 14^{* 2}$ \\
\hline Indexed ESV $\left(\mathrm{mL} / \mathrm{m}^{2}\right)$ & $27 \pm 9$ & $25 \pm 7$ & $15 \pm 5 * 3$ & $29 \pm 10$ & $51 \pm 21 * 2$ \\
\hline Indexed SV (mL/m²) & $50 \pm 11$ & $51 \pm 10$ & $40 \pm 7^{* 4}$ & $54 \pm 9$ & $58 \pm 13$ \\
\hline Indexed LV mass $\left(\mathrm{g} / \mathrm{m}^{2}\right)$ & $61 \pm 11 * 5$ & $70 \pm 9$ & $75 \pm 10$ & $108 \pm 24^{* 6}$ & $122 \pm 30 * 7$ \\
\hline Mass:volume ratio $(\mathrm{g} / \mathrm{mL})$ & $0.80 \pm 0.12 * 8$ & $0.92 \pm 0.10 * 9$ & $1.38 \pm 0.22 * 10$ & $1.39 \pm 0.38 * 11$ & $1.08 \pm 0.20$ \\
\hline \multicolumn{6}{|l|}{ T1-mapping } \\
\hline Native T1 (ms) & $1024 \pm 41$ & $1031 \pm 35$ & $1029 \pm 45$ & $1054 \pm 41 * 12$ & $1062 \pm 41 * 13$ \\
\hline Extracelluar volume fraction (\%) & $\ldots$ & $27 \pm 3$ & $26 \pm 3$ & $29 \pm 4 * 14$ & $30 \pm 3 * 15$ \\
\hline Myocardial cell volume fraction (\%) & $\ldots$ & $73 \pm 3$ & $74 \pm 3$ & $71 \pm 4^{* 14}$ & $70 \pm 3^{* 15}$ \\
\hline \multicolumn{6}{|l|}{ Circumferential myocardial function } \\
\hline Peak strain (\%) & $-17.4 \pm 2.6$ & $-17.6 \pm 3.0$ & $-17.1 \pm 3.2$ & $-15.5 \pm 3.1^{* 16}$ & $-12.8 \pm 4.6^{* 17}$ \\
\hline Peak systolic strain rate $(\% / s)$ & $-101 \pm 13$ & $-107 \pm 28$ & $-115 \pm 38$ & $-98 \pm 20 * 18$ & $-70 \pm 20 * 19$ \\
\hline Peak diastolic strain rate $(\% / s)$ & $101 \pm 26$ & $102 \pm 26$ & $90 \pm 24$ & $82 \pm 23 * 20$ & $65 \pm 21 * 21$ \\
\hline \multicolumn{6}{|l|}{ Aortic function } \\
\hline Compliance $\left(\mathrm{mm}^{2} / \mathrm{mm} \mathrm{Hg}\right)$ & $2.27 \pm 1.13^{* 22}$ & $1.62 \pm 1.21$ & $0.99 \pm 0.70$ & $1.60 \pm 1.09$ & $1.27 \pm 0.72$ \\
\hline Distensibility $\left(\mathrm{mm}^{2} / \mathrm{mm} \mathrm{Hg} \times 10^{3}\right)$ & $3.48 \pm 2.14^{* 23}$ & $2.26 \pm 1.71$ & $1.22 \pm 0.82 * 24$ & $1.84 \pm 1.46$ & $1.28 \pm 0.72$ \\
\hline \multicolumn{6}{|c|}{ 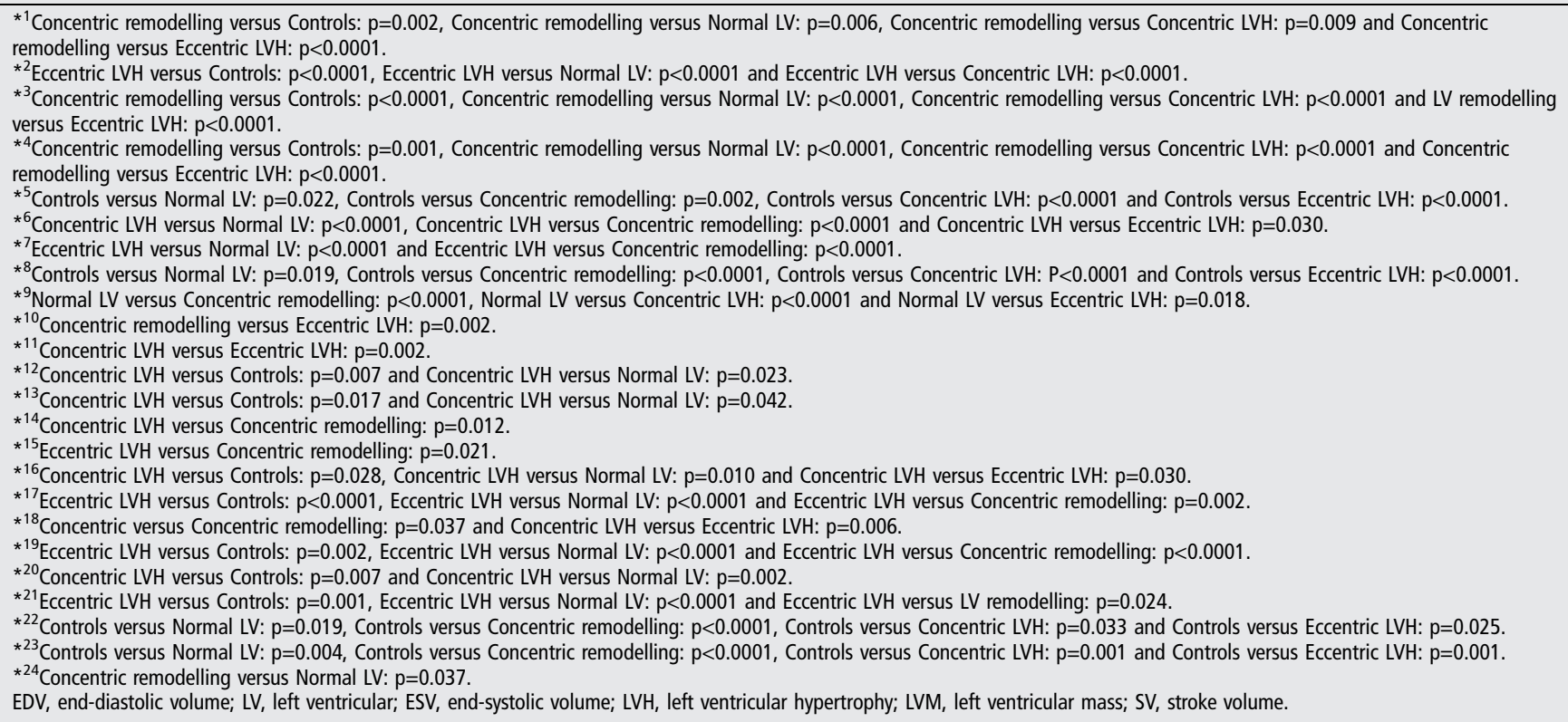 } \\
\hline
\end{tabular}

association with increased myocardial interstitial fibrosis or myocardial systolic strain impairment relative to controls, but these subjects demonstrated the most aortic stiffness.

\section{Left ventricular hypertrophy}

Hypertensive LVH was associated with significantly elevated native T1 compared with normotensive controls and significantly elevated native T1 and ECV compared with hypertensive subjects without LVH, which is consistent with work by Kuruvilla et $a l^{18}$ and Treibel et $a l^{19}$ in their studies of 43 and 40 hypertensive subjects, respectively. Our larger sample size of 88 hypertensive subjects may provide further insights into the pathophysiology of the spectrum of hypertensive heart disease. Concentric and eccentric hypertensive LVH are associated with adverse cardiovascular prognosis. ${ }^{6}$ We show that the eccentric form of LVH is associated with most advanced intracellular and interstitial myocardial expansion.

We also explored the functional implications of hypertensive LVH with CMR myocardial strain analysis. Eccentric and concentric LVH subgroups exhibited significant circumferential strain impairment compared with other hypertensive phenotypes. Our findings are consistent with previous echocardiographic studies. ${ }^{20}$ However, we additionally demonstrate that both increasing indexed myocardial cell and interstitial volume correlate significantly with worsening circumferential strain values. A putative mechanism to explain the relationship between strain and interstitial expansion is that LV stiffness increases with increased interstitial fibrosis, culminating in reduced end-diastolic muscle fibre length and, in turn, reduced myocardial contraction and LV systolic strain. ${ }^{21}$ The relationship 
Table 4 T1-mapping, myocardial strain and aortic function data corrected for covariates* for hypertensive subjects

\begin{tabular}{|c|c|c|c|c|}
\hline & \multicolumn{3}{|c|}{ Hypertensive subjects $(n=88)$} & \\
\hline & \multicolumn{2}{|c|}{ Normal indexed LVM ( $n=56)$} & \multicolumn{2}{|c|}{ Elevated indexed LVM $(n=32)$} \\
\hline & Normal LV ( $n=41)$ & Concentric remodelling ( $n=15)$ & Concentric LVH ( $n=24)$ & Eccentric LVH $(n=8)$ \\
\hline \multicolumn{5}{|l|}{ T1-mapping } \\
\hline Native T1 (ms) & $1031 \pm 6$ & $1025 \pm 10$ & $1054 \pm 8^{* 1}$ & $1067 \pm 15^{\star 2}$ \\
\hline Extracellular volume fraction (\%) & $27 \pm 1$ & $26 \pm 1$ & $29 \pm 1 * 3$ & $30 \pm 1 * 4$ \\
\hline \multicolumn{5}{|l|}{ Circumferential myocardial function } \\
\hline Peak strain $(\%)$ & $-16.9 \pm 0.5$ & $-17.4 \pm 0.8$ & $-16.1 \pm 0.6$ & $-14.2 \pm 1.1 * 5$ \\
\hline Peak systolic strain rate $(\% / \mathrm{s})$ & $-104 \pm 4$ & $-120 \pm 7$ & $-99 \pm 5 * 6$ & $-76 \pm 10 * 7$ \\
\hline Peak diastolic strain rate $(\% / s)$ & $95 \pm 4$ & $97 \pm 6$ & $85 \pm 5$ & $80 \pm 8$ \\
\hline \multicolumn{5}{|l|}{ Aortic function } \\
\hline Compliance $\left(\mathrm{mm}^{2} / \mathrm{mm} \mathrm{Hg}\right)$ & $1.61 \pm 0.19$ & $0.93 \pm 0.28^{* 8}$ & $1.73 \pm 0.23$ & $1.47 \pm 0.40$ \\
\hline Distensibility $\left(\mathrm{mm}^{2} / \mathrm{mm} \mathrm{Hg} \times 10^{3}\right)$ & $2.27 \pm 0.26$ & $1.05 \pm 0.39 * 9$ & $2.04 \pm 0.30$ & $1.57 \pm 0.55$ \\
\hline \multicolumn{5}{|c|}{$\begin{array}{l}\text { "Multiple linear regression accounting for the covariates of age, gender, body mass index, diabetes, office systolic } b \\
\text { antihypertensive medications. Data are presented as mean } \pm \text { SE. } \\
{ }^{* 1} \text { Concentric LVH versus normal LV: } p=0.033 \text { and concentric LVH versus concentric remodelling: } p=0.028 \text {. } \\
{ }^{2} \text { Eccentric LVH versus normal LV: } p=0.031 \text { and eccentric LVH versus concentric remodelling: } p=0.018 \text {. } \\
{ }^{* 3} \text { Concentric LVH versus normal LV: } p=0.013 \text { and concentric LVH versus concentric remodelling: } p=0.001 \text {. } \\
{ }^{4} \text { Eccentric LVH versus normal LV: } p=0.022 \text { and eccentric LVH versus concentric remodelling: } p=0.001 \text {. } \\
{ }^{5} \text { Eccentric LVH versus normal LV: } p=0.047 \text { and eccentric LVH versus concentric remodelling: } p=0.024 \text {. } \\
{ }^{66} \text { Concentric LVH versus concentric remodelling: } p=0.025 \text { and concentric LVH versus eccentric LVH: } p=0.038 \text {. } \\
{ }^{* 7} \text { Eccentric LVH versus normal LV: } p=0.016 \text { and eccentric LVH versus concentric remodelling: } p=0.002 \text {. } \\
{ }^{* 8} \text { Concentric remodelling versus concentric LVH: } p=0.028 \text {. } \\
{ }^{* 9} \text { Concentric remodelling versus normal LV: } p=0.020 \text { and concentric remodelling versus concentric LVH: } p=0.048 \text {. } \\
\text { LV, left ventricular; LVH, left ventricular hypertrophy; LVM, left ventricular mass. }\end{array}$} \\
\hline
\end{tabular}

between myocardial cell volume with strain may be explained by the fact that there is no significant change in external LV diameter during systole $\mathrm{e}^{22}$ and conservation of myocardial volume, with only negligible capillary bed compression, over the cardiac cycle. Consequently, in a hypertrophied LV with elevated end-diastolic wall thickness, less endocardial displacement (the output from myocardial strain) may be required to achieve the same stroke volume. ${ }^{23}$

\section{Concentric remodelling and LVH: a spectrum or distinct entities?}

Hypertensive patients with normal indexed LVM may have normal LV structure (with normal M/V) or concentric remodelling (with elevated $\mathrm{M} / \mathrm{V}$ ). The latter is associated with adverse prognosis. ${ }^{6}$ Interestingly, in our study, hypertensive subjects with concentric remodelling had no intracellular or extracellular myocardial expansion compared with normotensives and no evidence of significant myocardial systolic strain dysfunction. The lack of strong or significant positive correlation between SBP or DBP and native T1 or ECV suggests the development of myocardial fibrosis in hypertensive heart disease in our cohort is not simply linearly related to arterial pressure. Excess sympathetic activity may drive myocardial changes independently of $\mathrm{BP}^{24}$ Myocardial mechanical stress in response to pressure overload can upregulate pro-fibrotic and pro-hypertrophic genetic pathways $^{25}$ and may also be implicated. It is possible the reduction in EDV is a compensatory mechanism to 'unload' the pressureloaded $\mathrm{LV}^{4}$

Concentric remodelling subjects had increased aortic stiffness. Age and female gender are associated with increased aortic stiffness. ${ }^{26}$ Although our concentric remodelling cohort were significantly older than controls and hypertensive subjects with
A

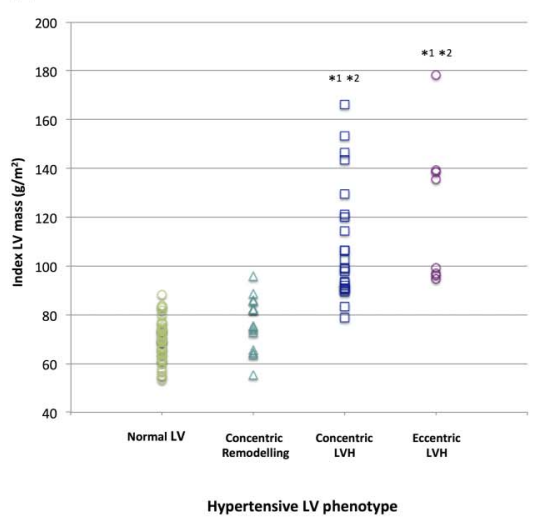

B

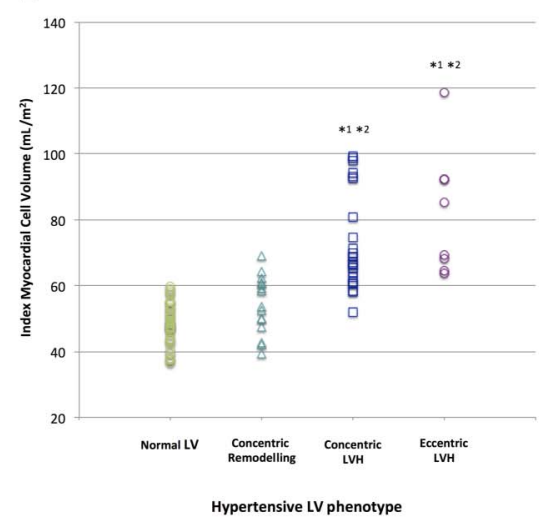

C

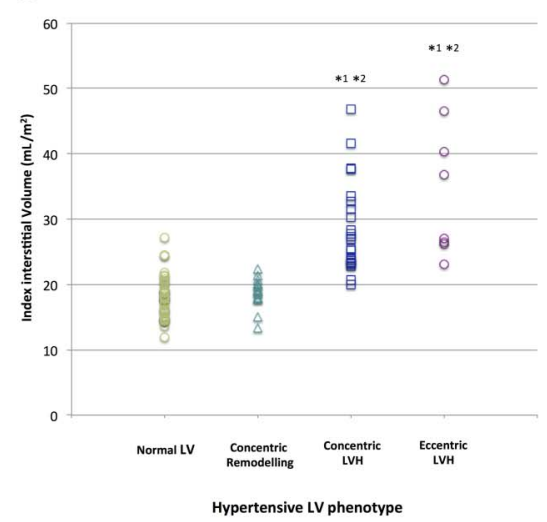

Figure 2 Dotplots showing differences in (A) indexed LV mass, $(B)$ indexed myocardial cell volume and (C) indexed interstitial volume between hypertensive LV phenotypes. ${ }^{* 1}$ Versus Normal LV: $p<0.0001,{ }^{* 2}$ Versus Concentric remodelling: $p<0.0001$. LV, left ventricular; LVH, left ventricular hypertrophy. 

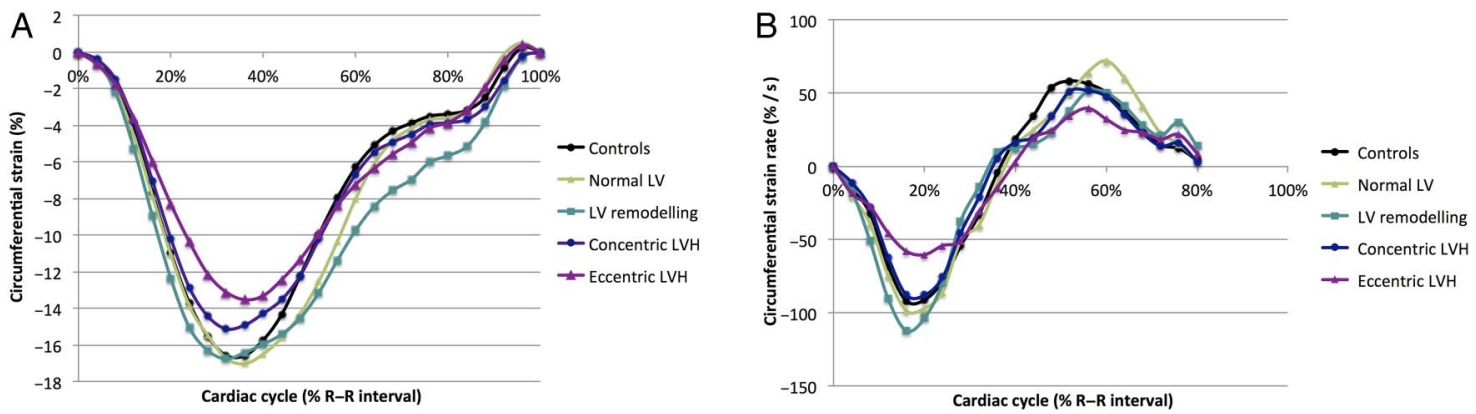

Figure 3 (A) Mean circumferential strain of the mid-myocardium over the cardiac cycle (B) Mean circumferential strain rate of the mid-myocardium over the cardiac cycle. LV, left ventricular; LVH, left ventricular hypertrophy.

normal LV structure, there were no significant differences in age or gender between concentric remodelling and concentric LVH or eccentric LVH. Furthermore, differences in aortic distensibility persisted after correcting for the covariates of age and gender. Severity of elevated BP (office BP and prevalence of ESH/ESC grade 3 hypertension) was not different between concentric remodelling and $\mathrm{LVH}$, but we were not able to investigate the impact of time spent at elevated BPs in the current study, which may be implicated. The interaction with the autonomic nervous system may, again, be important. Sympathetic neural mechanisms may have an arterial stiffening effect. Carotid-femoral pulse wave velocity, a marker of aortic stiffness, has been demonstrated to be linked to muscle sympathetic nerve activity (MSNA) in human subjects. ${ }^{27}$ The degree of MSNA can vary among hypertensive subjects. A putative explanation is that subjects with high MSNA have resultant increased aortic stiffness, and then the left ventricle remodels in response. Further longitudinal cohort studies are required to determine whether LV remodelling occurs in response to hypertension or whether it predates and is implicated in the aetiology of the hypertension.

\section{Treatment implications}

Hypertension is the strongest modifiable risk factor for cardiac morbidity and mortality. ${ }^{1}$ LVH can regress with appropriate antihypertensive treatment. However, a risk of heart failure persists after $\mathrm{LVH}$ regression, ${ }^{28}$ suggesting that current treatment strategies fail to tackle adverse myocardial changes beyond LVM. In a mouse model of hypertensive LVH, antihypertensive treatment caused interstitial fibrosis and cardiomyocyte hypertrophy regression. ${ }^{29}$ However, there has been failure to translate significant benefits of antifibrotic type therapies into patients with heart failure with preserved ejection fraction, who are often elderly and have long-standing hypertension. Large-scale human studies will be required to assess whether regression in myocyte mass and/or interstitial fibrosis occur with targeted antihypertensive agents and to categorically determine their relative importance with regard to: (i) regain of regional myocardial functional and (ii) improvement in overall cardiovascular prognosis. Such studies would help clarify whether myocardial interstitial fibrosis in hypertensive heart disease represents a viable therapeutic target. Understanding why altered aortic function occurs more in certain hypertensive phenotypes may have important treatment implications too; for example, antihypertensive agents with vasodilatory properties may be less effective in subjects with concentric remodelling and the stiffest aortas.

\section{Limitations}

Contrast medium was not administered to our normotensive control cohort. Consequently, there is no corresponding ECV data. However, the lack of significant difference between native T1 values between controls and hypertensive subjects with normal LV suggests the ECV is normal in this hypertensive subgroup, which essentially acts as hypertensive controls.

Our sample size of 88 hypertensive subjects is modest but represents the largest study of T1-mapping in hypertensive subjects until now. Nevertheless, we were unable to determine the impact of hypertension duration, or antihypertensive treatment strategies, on the variables investigated.

We do not have prognostic data in our cohort due to the short follow-up time and overall low event rate but prognostic data related to LV geometry are well established from large prospective, population-based studies. ${ }^{6}$

The voxel-tracking software used to generate strain values makes assumptions regarding conservation of myocardial mass over the cardiac cycle to derive strain values. Contemporaneous
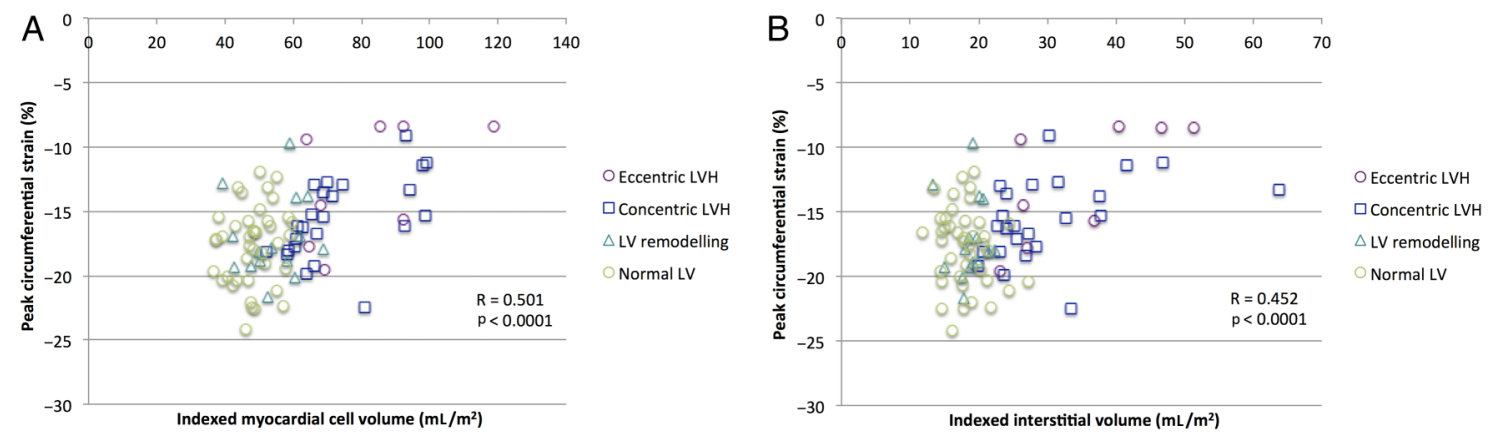

Figure 4 Peak circumferential strain versus $(A)$ indexed myocardial cell volume $(R=0.501, p<0.0001)$ and versus $(B)$ indexed interstitial volume $(R=0.452, p<0.0001)$. LV, left ventricular; LVH, left ventricular hypertrophy. 
echocardiographic data were not available in all subjects, but have previously been investigated. ${ }^{19}$

\section{Conclusion}

In hypertensive heart disease, structural differences exist at the intracellular/extracellular myocardial level across hypertensive phenotypes and are associated with functional consequences. Concentric and eccentric LVH are associated with significant intracellular and interstitial expansion with significant systolic and diastolic strain impairment. Concentric remodelling is associated with normal intracellular/extracellular myocardial structure and function but increased aortic stiffness. Our results may help explain why LVH, in particular eccentric LVH, has poor cardiovascular prognosis. Native T1 and myocardial ECV may become novel imaging biomarkers to characterise hypertensive heart disease and eventually help guide and monitoring treatment response with antifibrotic agents in certain hypertensive individuals.

\section{Key messages}

\section{What is already known on this subject?}

Hypertensive heart disease has a spectrum of left ventricular (LV) phenotypes that are associated with varying cardiovascular prognosis but the underlying pathophysiological mechanisms leading to these differences are incompletely understood.

\section{What might this study add?}

This study demonstrates differences in diffuse myocardial interstitial fibrosis, myocardial circumferential strain and aortic distensibility and compliance across the various hypertensive heart disease LV phenotypes, using multiparametric cardiovascular magnetic resonance (CMR). Our findings may help explain, at least in part, the differing cardiovascular prognosis observed in the different LV phenotypes.

\section{How might this impact on clinical practice?}

The non-invasive detection of subclinical abnormalities with CMR, such as the burden of diffuse myocardial interstitial and abnormal myocardial strain and aortic distensibility, may represent novel biomarkers in arterial hypertension to guide targeted pharmacological interventions in future.

\footnotetext{
Author affiliations

${ }^{1}$ NIHR Bristol Cardiovascular Biomedical Research Unit, Cardiac Magnetic Resonance Department, Bristol Heart Institute, University Hospitals Bristol NHS Foundation Trust, Bristol, UK

${ }^{2}$ School of Physiology, Pharmacology and Neurosciences, Faculty of Biomedical Sciences, University of Bristol, University Walk, Bristol, UK

${ }^{3}$ Department of Surgical, Microsurgical and Medical Sciences, Institute of Radiology, University of Sassari, Sassari, Piazza D'Armi, Province of Sassari, Italy

${ }^{4}$ Department of Cardiology, Bristol Royal Infirmary, University Hospitals Bristol NHS Foundation Trust, Bristol, UK

${ }^{5}$ Department of Cardiology, University Hospital of Wales, Cardiff

${ }^{6}$ Department of Clinical Radiology, Bristol Royal Infirmary, University Hospitals Bristol NHS Foundation Trust, Bristol, UK

${ }^{7}$ Department of General Medicine, Bristol Royal Infirmary, University Hospitals Bristol NHS Foundation Trust, Bristol, UK

${ }^{8}$ CardioNomics Research Group, Clinical Research Imaging Centre (CRIC) Bristol, Bristol Heart Institute, University Hospitals Bristol NHS Foundation Trust, Bristol, UK
}

Twitter Follow Jonathan Rodrigues at @JCLRodrigues
Acknowledgements This work was supported by the Bristol NIHR Cardiovascular Biomedical Research Unit at the Bristol Heart Institute. The views expressed are those of the authors and not necessarily those of the National Health Service (NHS), National Institute for Health Research or Department of Health. We thank Christopher Lawton, Superintendent Radiographer, and the Bristol Heart Institute CMR radiographers for their expertise in performing the CMRs. JCLR: Clinical Society of Bath Postgraduate Research Bursary 2014 and Royal College of Radiologists Kodak Research Scholarship 2014. ECH and JFRP are funded by the British Heart Foundation.

Contributors JCLR conceived and designed the study, acquired data, analysed data, drafted the manuscript and revised it critically for important intellectual content. AMA, AGD, GS, SL, CG, LEKR and AEB acquired data and revised the work for important intellectual content. ECH, MCKH, AKN and JFRP contributed to the interpretation of data and revised the work for important intellectual content. NEM and CB-D contributed to the design of the work, analysed data and revised the work for critically important intellectual content. All authors gave final approval of the version to be published. All authors agree to be accountable for all aspects of the work in ensuring that questions related to the accuracy or integrity of any part of the work are appropriately investigated and resolved. CB-D is the overall guarantor for the work.

Funding Bristol NIHR Cardiovascular Biomedical Research Unit in The Bristol Heart Institute, University of Bristol and University Hospitals Bristol NHS Foundation Trust.

Competing interests CB-D is a consultant for Circle Cardiovascular Imaging.

\section{Patient consent Obtained.}

Ethics approval NHS research ethics committee.

Provenance and peer review Not commissioned; externally peer reviewed.

Open Access This is an Open Access article distributed in accordance with the Creative Commons Attribution Non Commercial (CC BY-NC 4.0) license, which permits others to distribute, remix, adapt, build upon this work non-commercially, and license their derivative works on different terms, provided the original work is properly cited and the use is non-commercial. See: http://creativecommons.org/ licenses/by-nc/4.0/

\section{REFERENCES}

1 Haider AW, Larson MG, Benjamin EJ, et al. Increased left ventricular mass and hypertrophy are associated with increased risk for sudden death. J Am Coll Cardiol 1998:32:1454-9.

2 Kannel WB, Castelli WP, McNamara PM, et al. Role of blood pressure in the development of congestive heart failure. The Framingham study. N Engl J Med 1972;287:781-7.

3 Mancia G, Fagard R, Narkiewicz K, et al. 2013 ESH/ESC guidelines for the management of arterial hypertension: the Task Force for the Management of Arterial Hypertension of the European Society of Hypertension (ESH) and of the European Society of Cardiology (ESC). Eur Heart J 2013;34:2159-219.

4 Ganau A, Devereux RB, Roman MJ, et al. Patterns of left ventricular hypertrophy and geometric remodeling in essential hypertension. J Am Coll Cardiol 1992;19:1550-8.

5 Dweck MR, Joshi S, Murigu T, et al. Left ventricular remodeling and hypertrophy in patients with aortic stenosis: insights from cardiovascular magnetic resonance. J Cardiovasc Magn Reson 2012;14:50.

6 Krumholz HM, Larson M, Levy D. Prognosis of left ventricular geometric patterns in the Framingham Heart Study. J Am Coll Cardiol 1995;25:879-84.

7 Querejeta R, Varo N, López B, et al. Serum carboxy-terminal propeptide of procollagen type I is a marker of myocardial fibrosis in hypertensive heart disease. Circulation 2000;101:1729-35.

8 Flett AS, Hayward MP, Ashworth MT, et al. Equilibrium contrast cardiovascular magnetic resonance for the measurement of diffuse myocardial fibrosis: preliminary validation in humans. Circulation 2010;122:138-44.

9 Miller CA, Naish JH, Bishop P, et al. Comprehensive validation of cardiovascular magnetic resonance techniques for the assessment of myocardial extracellular volume. Circ Cardiovasc Imaging 2013;6:373-83.

10 Childs H, Ma L, Ma M, et al. Comparison of long and short axis quantification of left ventricular volume parameters by cardiovascular magnetic resonance, with ex-vivo validation. J Cardiovasc Magn Reson 2011;13:40.

11 Maceira AM, Prasad SK, Khan M, et al. Normalized left ventricular systolic and diastolic function by steady state free precession cardiovascular magnetic resonance. J Cardiovasc Magn Reson 2006;8:417-26.

12 Messroghli DR, Greiser A, Fröhlich M, et al. Optimization and validation of a fully-integrated pulse sequence for modified look-locker inversion-recovery (MOLLI) T1 mapping of the heart. J Magn Reson Imaging 2007;26:1081-6.

13 Pica S, Sado DM, Maestrini V, et al. Reproducibility of native myocardial T1 mapping in the assessment of Fabry disease and its role in early detection of cardiac involvement by cardiovascular magnetic resonance. J Cardiovasc Magn Reson 2014;16:99. 
14 Shah AS, Chin CW, Vassiliou V, et al. Left ventricular hypertrophy with strain and aortic stenosis. Circulation 2014;130:1607-16.

15 Flett AS, Sado DM, Quarta G, et al. Diffuse myocardial fibrosis in severe aortic stenosis: an equilibrium contrast cardiovascular magnetic resonance study. Eur Heart J Cardiovasc Imaging 2012;13:819-26.

16 Bistoquet A, Oshinski J, Skrinjar O. Myocardial deformation recovery from cine MRI using a nearly incompressible biventricular model. Med Image Anal 2008;12:69-85.

17 Groenink M, de Roos A, Mulder BJ, et al. Biophysical properties of the normal-sized aorta in patients with Marfan syndrome: evaluation with MR flow mapping. Radiology 2001;219:535-40.

18 Kuruvilla $\mathrm{S}$, Janardhanan $\mathrm{R}$, Antkowiak $\mathrm{P}$, et al. Increased extracellular volume and altered mechanics are associated with LVH in hypertensive heart disease, not hypertension alone. JACC Cardiovasc Imaging 2015;8:172-80.

19 Treibel TA, Zemrak F, Sado DM, et al. Extracellular volume quantification in isolated hypertension —changes at the detectable limits? I Cardiovasc Magn Reson 2015;17:74.

20 Mizuguchi Y, Oishi Y, Miyoshi H, et al. Concentric left ventricular hypertrophy brings deterioration of systolic longitudinal, circumferential, and radial myocardial deformation in hypertensive patients with preserved left ventricular pump function. J Cardiol 2010;55:23-33.

21 McLenachan JM, Dargie HJ. Ventricular arrhythmias in hypertensive left ventricular hypertrophy. Relationship to coronary artery disease, left ventricular dysfunction, and myocardial fibrosis. Am J Hypertens 1990;3:735-40.
22 Emilsson $\mathrm{K}$, Brudin L, Wandt B. The mode of left ventricular pumping: is there an outer contour change in addition to the atrioventricular plane displacement? Clin Physiol 2001;21:437-46.

23 Maclver DH, Adeniran I, Zhang $\mathrm{H}$. Left ventricular ejection fraction is determined by both global myocardial strain and wall thickness. IJC Hear Vasc 2015;7: 113-18.

24 Seravalle G, Lonati L, Buzzi S, et al. Sympathetic nerve traffic and baroreflex function in optimal, normal, and high-normal blood pressure states. J Hypertens 2015;33:1411-17.

25 Adams JW, Sakata Y, Davis MG, et al. Enhanced Galphaq signaling: a common pathway mediates cardiac hypertrophy and apoptotic heart failure. Proc Natl Acad Sci USA 1998;95:10140-5.

26 Nethononda RM, Lewandowski AJ, Stewart R, et al. Gender specific patterns of age-related decline in aortic stiffness: a cardiovascular magnetic resonance study including normal ranges. J Cardiovasc Magn Reson 2015;17:20.

27 Swierblewska E, Hering D, Kara T, et al. An independent relationship between muscle sympathetic nerve activity and pulse wave velocity in normal humans. $J$ Hypertens 2010;28:979-84.

28 Devereux RB, Wachtell K, Gerdts E, et al. Prognostic significance of left ventricular mass change during treatment of hypertension. JAMA 2004;292:2350-6.

29 Coelho-Filho OR, Shah RV, Neilan TG, et al. Cardiac magnetic resonance assessment of interstitial myocardial fibrosis and cardiomyocyte hypertrophy in hypertensive mice treated with spironolactone. J Am Heart Assoc 2014;3 e000790-e000790. 